\title{
Chamado à ação de São Paulo para prevenção e controle da hipertensão arterial, 2020*
}

\author{
Norm RC Campbell', Aletta E Schutte ${ }^{2}$, Cherian V Varghese ${ }^{3}$, Pedro Ordunez ${ }^{4}$, Xin-Hua Zhang ${ }^{5}$, \\ Taskeen Khan ${ }^{3}$, James E Sharman ${ }^{6}$, Paul K Whelton', Gianfranco Parati ${ }^{8}$, Michael A Weber ${ }^{9}$, Marcelo \\ Orías $^{10}$, Marc G Jaffe ${ }^{11}$, Andrew E Moran ${ }^{12}$, Frida Liane Plavnik ${ }^{13}$, Venkata S Ram ${ }^{14}$, Michael Brainin ${ }^{15}$, \\ Mayowa O Owolabi ${ }^{16}$, Agustin J Ramirez ${ }^{17}$, Eduardo Barbosa ${ }^{18}$, Luiz Aparecido Bortolotto ${ }^{19}$, \\ Daniel T Lackland ${ }^{20}$
}

Como citar

Campbell NRC, Schutte AE, Varghese CV, Ordunez P, Zhang XH, Khan T et al. Chamado à ação de São Paulo para prevenção e controle da hipertensão arterial, 2020. Rev Panam Salud Publica. 2021;45:e27. https://doi.org/10.26633/RPSP.2021.27

RESUMO

- Cerca de 1/4 dos adultos têm hipertensão arterial, que é o fator de risco isolado mais importante para morte (incluídas as mortes por cardiopatia e acidente vascular cerebral).

- Existem políticas eficazes que poderiam facilitar escolhas pessoais saudáveis para evitar a elevação da pressão arterial e, se plenamente implementadas, podem prevenir a ocorrência da hipertensão arterial.

- É fácil rastrear e tratar a hipertensão, MAS somente cerca de 50\% dos adultos hipertensos estão cientes de sua condição, e apenas cerca de 1 em cada 7 é tratado adequadamente.

- A prevenção e controle da hipertensão é o principal mecanismo de prevenção e controle das doenças não transmissíveis e um modelo para outros riscos de doenças não transmissíveis.

- Tratamentos eficazes com mudanças de estilo de vida e medicamentos poderiam prevenir e controlar a hipertensão arterial na maioria das pessoas se aplicados sistematicamente à população; as intervenções simples são viáveis em todos os ambientes e podem melhorar a atenção primária.

- É necessária a ação continuada e urgente a fim de obter mudanças efetivas nas políticas públicas e no sistema de saúde para prevenir e controlar a hipertensão arterial.

Palavras-chave Hipertensão; doenças cardiovasculares; saúde global; dieta hipossódica; acidente vascular cerebral.

\footnotetext{
* Tradução oficial em português do artigo original em inglês pela Organização Pan-Americana da Saúde, com a autorização da Wiley Periodicals. Em caso de discrepância, prevalece a versão original em inglês. Wiley não é responsável pela exactidão da tradução do original em inglês ou por quaisquer erros que este possa conter.

Cita do artigo original: Campbell NRC, Schutte AE, Varghese CV, et al. São Paulo call to action for the prevention and control of high blood pressure: 2020 . J Clin Hypertens. 2019;21:1744-1752. https://doi.org/10.1111/jch.13741

1 Departamento de Medicina, Departamento de Medicina, Fisiologia e Farmacologia e Ciências da Saúde da Comunidade, Instituto O'Brien de Saúde Pública e Instituto Cardiovascular Libin de Alberta, Universidade de Calgary, Calgary, Alberta, Canadá. $\bowtie$ Norm Campbell, ncampbel@ucalgary.ca

2 Unidade de Hipertensão e Doença Cardiovascular, Equipe de Pesquisa de Hipertensão na África (HART, na sigla em inglês), Universidade Noroeste, Potchefstroom, África do Sul

3 Departamento de Doenças Não Transmissíveis, Organização Mundial da Saúde, Genebra, Suíça

4 Departamento de Doenças Não Transmissíveis e Saúde Mental, Organização Pan-Americana da Saúde, Washington, D.C., Estados Unidos

5 Instituto Liga de Hipertensão de Pequim, Pequim, China

6 Instituto Menzies para Pesquisa Médica, Universidade da Tasmânia, Hobart, Tasmânia, Austrália

7 Departamento de Epidemiologia e Medicina, Centro de Ciências da Saúde da Universidade de Tulane, Nova Orleans, Estados Unidos
}

\footnotetext{
8 Departamento de Medicina e Cirurgia, Universidade de Milão-Bicocca e Departamento de Ciências Cardiovasculares, Neurais e Metabólicas, e Instituto Auxológico Italiano, IRCCS, Hospital San Luca, Milão, Itália

9 Divisão de Medicina Cardiovascular, Universidade Estadual de Nova York Centro Médico Downstate, Brooklyn, Nova York, Estados Unidos

10 Sanatorio Allende, y Universidade Nacional de Córdoba, Córdoba, Argentina

11 Resolve to Save Lives, Uma Iniciativa da Vital Strategies, Nova York, Estados Unidos e Kaiser Permanente do Norte da Califórnia, South San Francisco, Califórnia, Estados Unidos

12 Controle Mundial de Hipertensão, Resolve to Save Lives, Uma iniciativa da Vital Strategies, Nova York, Estados Unidos

13 Grupo de Hipertensão, Hospital Alemão Oswaldo Cruz; Instituto do Coração (InCor); Sociedade Brasileira de Hipertensão, São Paulo, Brasil

14 Escola de Medicina Sudoeste da Universidade do Texas, Dallas, Estados Unidos; Faculdade de Medicina e Hospitais Apollo, Hyderabad, Índia; Universidade Macquarie, Faculdade de Medicina e Ciências da Saúde, Sydney, Austrália; Liga Mundial de Hipertensão, Escritório Regional do Sudeste Asiático, Hyderabad, Índia

15 Universidade do Danúbio, Krems, Áustria

16 Centro de Excelência para Doenças Não Transmissíveis da Aliança de Universidades de Pesquisa Africana, Universidade de Ibadan, Nigéria

17 Unidade de Hipertensão Arterial e Doenças Metabólicas, Hospital Universitário, Fundação Favaloro, Buenos Aires, Argentina

18 Sociedade Latino-americana de Hipertensão, Porto Alegre, Brasil
} 


\section{INTRODUÇÃO}

\subsection{Prevalência da hipertensão arterial}

- Em 2010, entre 1,13 e 1,4 bilhão de pessoas tinham hipertensão arterial (definida como PA > 140/90 mmHg). (Disponível em inglês em: https://www.who.int/news-room/fact-sheets/detail/hypertension, consultado em 16 de junho de 2019) $)^{1}$.

- Nas sociedades industrializadas, a pressão arterial aumenta com a idade, de modo que cerca de 9 em 10 adultos que vivam até 80 anos de idade desenvolverão hipertensão².

- Cerca de 1 em cada 4 adultos tem hipertensão (> 140/90 $\mathrm{mm} \mathrm{Hg}$ ), incluindo $40 \%$ das pessoas com idade acima de 25 anos (disponível em inglês em: https: / /www.who.int/news-room/fact-sheets/detail/hypertension, consultado em 16 de junho de 2019; https://www.who.int/news-room/fact-sheets/detail/hypertension, consultado em 16 de junho de $2019)^{1,3}$.

- A definição de hipertensão como PA > 130/80 mmHg aumenta a prevalência em cerca de 1,5 vezes, para cerca de $50 \%$ dos adultos $^{4,5}$.

\subsection{Impacto da hipertensão arterial na saúde}

- A hipertensão arterial é o principal fator de risco isolado e evitável para morte no mundo, e o $3^{\circ}$ principal fator de risco para incapacidade segundo o estudo Carga Global de Doença ${ }^{6}$.

- Calcula-se que a hipertensão arterial tenha causado 10,4 milhões de mortes (quase 19\% do total de mortes) e 218 milhões de anos de vida ajustados por incapacidade $(8,7 \%$ do total de DALYs) em $2017^{6}$.

- Cerca de 70\% das mortes relacionadas à hipertensão arterial ocorrem em pessoas com pressão arterial > 140/90 $\mathrm{mmHg}$, e as demais $30 \%$ ocorrem em pessoas com pressão arterial subótima, que é inferior a $140 / 90 \mathrm{mmHg}^{7}$.

- Mais de 50\% dos casos de doenças cardíacas, acidente vascular cerebral e insuficiência cardíaca são causados por aumento da pressão arterial ${ }^{7,8}$.

- A hipertensão é o fator de risco isolado mais importante para acidente vascular cerebral isquêmico, bem como hemorrágico ${ }^{9,10}$

- A hipertensão causa mais de $40 \%$ das mortes em diabéticos ${ }^{11}$; também é um importante fator de risco para morte fetal e materna na gravidez, demência e insuficiência renal (sobretudo em pessoas de ascendência africana) e pode causar cegueira ${ }^{7,12,13,14}$.

\subsection{Impactos na população de países de baixa, média e alta renda}

- A hipertensão é um importante fator de risco para morte em países de baixa, média e alta renda 7,15

- Dois terços das pessoas com hipertensão estão em países de baixa e média renda, onde vive a maior parte da população mundial (disponível em inglês em: https://www.who.int/ news-room/fact-sheets/detail/hypertension, consultado em 16 de junho de 2019) $)^{1,3}$.

\footnotetext{
19 Hospital das Clínicas da Universidade de São Paulo; Instituto do Coração (InCor); e Sociedade Brasileira de Hipertensão, São Paulo, Brasil
}

- O número de adultos hipertensos aumentou de 594 milhões em 1975 para 1,13 a 1,4 bilhão, e a maior parte desse aumento ocorreu em países de baixa e média renda (disponível em inglês em: https://www.who.int/news-room/fact-sheets / detail/hypertension, consultado em 16 de junho de 2019).

- A pressão arterial sistólica parece estar aumentando ao longo do tempo no leste, sudeste e sul da Ásia, na Oceana e na África subsaariana, enquanto vem diminuindo em outras regiões ${ }^{15}$

- A doença cardíaca e o acidente vascular cerebral ocorrem em pessoas mais jovens nos países de baixa e média renda ${ }^{1,3}$.

\subsection{Impacto econômico da hipertensão arterial}

- Cerca de 10\% dos gastos mundiais com atenção à saúde estão diretamente relacionados com a hipertensão arterial e suas complicações, como doença isquêmica do coração, insuficiência cardíaca e acidente vascular cerebral ${ }^{16}$.

- Cerca de 25\% dos gastos com atenção à saúde na Europa oriental e na Ásia central devem-se a doenças relacionadas com a pressão arterial ${ }^{16}$.

- O Plano de trabalho mundial para as doenças não transmissíveis (DNT) calcula que o manejo clínico dos riscos cardiovasculares, que inclui a hipertensão arterial, tenha um retorno de mais de três dólares para cada dólar investido ${ }^{17}$.

\section{FATORES DETERMINANTES DA HIPERTENSÃO ARTERIAL}

\subsection{Ambientes não saudáveis têm um papel importante no aumento da pressão arterial}

- Os ambientes não saudáveis são um importante fator de contribuição para estilos de vida e comportamentos não saudáveis.

- Há ampla variação nacional na prevalência de riscos para o surgimento da hipertensão arterial (p. ex., riscos alimentares, sedentarismo, obesidade, consumo excessivo de álcool), que provavelmente são os principais determinantes das diferenças nas taxas de prevalência de hipertensão. ${ }^{6}$

- A alimentação não saudável (excluindo o impacto alimentar sobre a obesidade) contribui para cerca de metade do número de pacientes com hipertensão arterial ${ }^{18}$.

- Cerca de 30\% dos casos de hipertensão arterial estão relacionados ao maior consumo de sal, e cerca de $20 \%$ estão relacionados à baixa ingestão de potássio (baixo consumo de frutas e hortaliças) $)^{18,19}$.

- A elevada proporção entre gorduras saturadas e ácidos graxos poli-insaturados também contribui para a hipertensão arterial ${ }^{20}$.

- O sedentarismo está relacionado a cerca de $20 \%$ dos casos de hipertensão arterial $^{18}$.

- A obesidade está associada a cerca de 30\% dos casos de hipertensão arterial ${ }^{18}$.

- O consumo excessivo de álcool também aumenta a pressão arterial e causa hipertensão arterial ${ }^{18}$.

- A vida sem tabaco é particularmente importante para as pessoas com hipertensão arterial, para prevenção das doenças cardiovasculares e outras doenças não transmissíveis ${ }^{3}$.

- A desigualdade educacional e socioeconômica tem um im-

20 Divisão de Neurociências Translacionais e Estudos Populacionais, Universidade de Medicina da Carolina do Sul, Charleston, Estados Unidos 
pacto significativo sobre a prevalência e o controle da hipertensão arterial ${ }^{21-23}$.

- Os governos aprovaram nove metas voluntárias mundiais com a finalidade abrangente de reduzir em $25 \%$ as mortes prematuras pelas quatro principais DNTs até 2025. As metas relacionadas com a hipertensão arterial e seus fatores de risco incluem os objetivos, para 2025, de redução de $25 \%$ do descontrole da pressão arterial, redução de 30\% da quantidade de sódio na alimentação, redução de $10 \%$ da atividade física insuficiente, redução de $30 \%$ do tabagismo a partir dos 15 anos de idade e cessação do crescimento da obesidade e da diabetes ${ }^{24}$.

\subsection{As intervenções clínicas para o controle da hipertensão arterial não têm sido aplicadas sistematicamente na maioria dos países}

- Em todo o mundo, cerca de metade dos adultos hipertensos desconhece que sua pressão arterial está aumentada, uma situação mais dramática em países de baixa renda ${ }^{1,25}$.

- Alguns dos que sabem ser hipertensos permanecem sem tratamento. Mesmo quando tratados, o controle da pressão arterial é insatisfatório na maioria ${ }^{1}$.

- Em países de alta renda, as taxas médias de conhecimento, tratamento e controle são respectivamente de $67 \%, 55,6 \%$ e $28,4 \%$, enquanto em países de baixa e média renda, as taxas são respectivamente de $37,9 \%, 29 \%$ e $7,7 \%, 1,26$.

- No âmbito mundial, menos de 1 em cada 5 adultos hipertensos tem a pressão controlada; na África subsaariana, essa proporção é inferior a 1 em 14 (disponível em inglês em: https: / / www.who.int/news-room/fact-sheets/detail/hypertension, consultado em 16 de junho de 2019) ${ }^{27}$.

\section{O QUE DÁ RESULTADO?}

\subsection{Investimentos em prevenção costumam reduzir custos}

- As intervenções políticas populacionais para criar ambientes de melhoria da alimentação e da atividade física costumam reduzir os custos e facilitar que as pessoas façam escolhas saudáveis ${ }^{28,29}$.

- Calcula-se que a redução de sódio na dieta propicie um retorno de 13 a 18 dólares para cada dólar investido ${ }^{17,30}$.

- As políticas recomendadas de prevenção ou manejo das DNTs, aí incluída a hipertensão arterial, abrangem melhoria da alimentação, aumento da atividade física, evitar o tabagismo e redução do uso prejudicial de álcool são previstas pela Organização Mundial da Saúde ${ }^{31,32,33}$ (disponível em inglês em: https://www.who.int/tobacco/mpower/publications / en/, consultado em 7 de maio de 2019).

\subsection{Investimentos em tratamento e controle são custo-efetivos}

- O tratamento efetivo da pressão arterial > 140/90 mmHg reduz acidentes vasculares cerebrais e doenças cardíacas ${ }^{34}$.

- O tratamento efetivo da pressão arterial > 130/80 mmHg reduz acidentes vasculares cerebrais e doenças cardíacas nas pessoas com risco cardiovascular moderado a alto (risco > $10-19 \%$ de desenvolver doença cardiovascular nos próximos
10 anos, nos casos de risco moderado, e risco $>20 \%$ de desenvolver doença cardiovascular nos próximos 10 anos, nos casos de alto risco) ${ }^{34}$.

- Nos Estados Unidos, Canadá e Finlândia, mais de 80\% dos hipertensos têm outros riscos cardiovasculares e/ou evidências de lesão relacionada com a pressão arterial (doença cardíaca, acidente vascular cerebral ou lesão renal ${ }^{35,36}$.

- A avaliação objetiva de risco cardiovascular absoluto pode ser útil no manejo geral dos pacientes, incluindo a possível necessidade de menor limiar de tratamento e de meta da pressão arterial, bem como de tratamento da dislipidemia ${ }^{34,37-39}$.

- O tratamento da hipertensão arterial é fundamental na rotina de prevenção primária das DNTs, o que justifica a verificação periódica da pressão arterial de todos os adultos e o tratamento dos hipertensos.

- Os resultados do estudo SPRINT e uma nova análise do estudo ACCORD enfatizam que, em geral, o tratamento intensivo da hipertensão arterial é benéfico 1 na redução da mortalidade de pessoas com alto risco de doença cardiovascular, embora o tratamento intensivo da pressão arterial possa causar efeitos colaterais ${ }^{40,41}$.

\section{QUAIS SÃO AS BARREIRAS?}

\subsection{Inércia política}

- Muitos países não implementaram políticas públicas amplas e efetivas que resultariam em condições ideais de alimentação e atividade física, controle do consumo de bebidas alcoólicas e do tabagismo, bem como prevenção de muitas doenças não transmissíveis, incluindo riscos como a hipertensão arterial $^{33}$ [disponível em https: / / www.wcrf.org/int/policy / nourishing-database (em inglês), consultado em 17 de abril de 2019; https:/ /www.who.int/es/news-room/fact-sheets / detail/physical-activity (em espanhol), consultado em 5 de maio de 2019; e https://www.who.int/substance_abuse/ activities /fadab/msb_adab_gas_progress_report.pdf?ua=1 (em inglês), consultado em 5 de maio de 2019].

- Muitos países não ampliaram a atenção primária para abordar as necessidades de saúde da população, incluindo o diagnóstico e manejo da hipertensão arterial ${ }^{42,43}$.

- Muitas organizações e sociedades cardiovasculares e de hipertensão nacionais não publicaram planos estratégicos para prevenção, diagnóstico, tratamento e controle da hipertensão arterial e não defendem efetivamente políticas alinhadas com aquelas elaboradas pela OMS para a prevenção e o controle efetivos das DNTs, incluindo a hipertensão arterial.

\subsection{Inércia dos sistemas de saúde}

- Embora a melhora do controle da hipertensão arterial seja uma das intervenções clínicas mais eficazes e custo-efetivas para reduzir a carga de doenças não transmissíveis e atender os objetivos de desenvolvimento sustentável (ODS), a prevenção e o controle da hipertensão arterial não estão entre as prioridades máximas políticas e de saúde em muitos países ${ }^{44}$. A maioria dos países não tem uma orientação estratégica de saúde pública e de sistemas de saúde para o controle da hipertensão ${ }^{45}$.

- A orientação estratégica de saúde pública/ sistemas de saúde para o controle da hipertensão arterial abrange a cobertura de toda a população para: acesso fácil a um suprimento, con- 
fiável e a preços módicos, de um conjunto básico de medicamentos anti-hipertensivos de alta qualidade, incluindo combinações em comprimido único; fácil acesso à atenção com base em trabalho de equipe; medição/avaliação sistemática da pressão arterial em todas as consultas médicas; rastreamento comunitário da hipertensão arterial estreitamente vinculado ao sistema de saúde; uso de monitores eletrônicos de pressão arterial validados por pessoal treinado recentemente; uso de um algoritmo simples de diagnóstico e tratamento; uso de um registro de hipertensão arterial com relatório de desempenho; e levantamentos populacionais periódicos de hipertensão (3-5 anos) $)^{46-52}$.

- Muitos profissionais de saúde não iniciam o tratamento ou não ajustam adequadamente a dose do medicamento em pessoas com medidas elevadas da pressão arterial ${ }^{53,54}$.

\section{UMA AGENDA DE TRANSFORMAÇÃO PARA PREVENÇÃO E CONTROLE DA HIPERTENSÃO ARTERIAL}

- Políticas e intervenções transformadoras do serviço de saúde podem melhorar os serviços de atenção primária à saúde e o controle da hipertensão arterial e são altamente recomendadas pela Organização Mundial da Saúde, iniciativa Resolve to Save Lives, Sociedade Internacional de Hipertensão, Liga Mundial de Hipertensão e outras organizações.

- A Organização Mundial da Saúde liderou diversas organizações internacionais no desenvolvimento de recursos de ponta que traçam políticas e intervenções para prevenção e controle das doenças cardiovasculares e da diabetes. $\mathrm{O}$ mais relevante e atualizado é o pacote de medidas técnicas HEARTS (disponível em inglês em: https://www.who.int/cardiovascular_diseases/hearts/en/, consultado em 17 de abril de 2019).

- A iniciativa Resolve to Save Lives (RTSL), da Vital Strategies, é um programa mundial filantrópico para melhorar o controle da hipertensão arterial, reduzir o sódio na alimentação, eliminar as gorduras trans artificiais dos alimentos e reforçar os preparativos para uma pandemia (disponível em inglês em: https://www.resolvetosavelives.org/, consultado em 17 de abril de 2019).

- Para o controle da hipertensão arterial, o pacote de medidas técnicas HEARTS defende fortemente o aumento da capacidade clínica da atenção primária, por meio de atenção baseada em trabalho de equipe, suprimento confiável e acessível de medicamentos anti-hipertensivos de qualidade (incluindo combinações em comprimido único quando apropriado), medição/avaliação sistemática da pressão arterial em todas as consultas, uso de monitore de pressão arterial eletrônicos validados por pessoal treinado recentemente, uso de um algoritmo simples de diagnóstico e tratamento, uso de um registro de hipertensão com relatório de desempenho, além de levantamentos populacionais periódicos da hipertensão arterial.

- A Sociedade Internacional de Hipertensão promove todos os anos, com organizações parceiras, o programa Maio, Mês de Medição para rastreamento da pressão arterial por meio de técnicas padronizadas e a conscientização sobre hipertensão arterial; e a Liga Mundial de Hipertensão promove anualmente, com organizações parceiras, o Dia Mundial da Hipertensão (17 de maio) para aumentar a conscientização sobre hipertensão $\operatorname{arterial}^{25,55-57}$.

\section{UMA ESTRATÉGIA CLÍNICA TRANSFORMADORA}

\subsection{Profissionais e estabelecimentos de saúde e organizações de atenção à saúde}

- Permanecer atualizado com relação aos melhores padrões mundiais para prevenção e controle da hipertensão arterial.

- Medir a pressão arterial em todas as consultas apropriadas.

- Promover, adquirir e impor o uso de monitores automáticos e validados para avaliação exata da pressão arterial.

- Desenvolver programas padronizados de treinamento para pacientes e profissionais de saúde quanto à medição correta da pressão arterial.

- Garantir que os responsáveis pela medição da pressão arterial sejam treinados e avaliados periodicamente para assegurar a exatidão dos valores pressóricos obtidos. Exigir cursos de certificação nos lugares onde estiverem disponíveis.

- Avaliar o risco cardiovascular, com auxílio de um método objetivo (programa de computador, tabela de risco), e a presença de doença cardiovascular ou lesão de órgão-alvo relacionada com a hipertensão (p. ex., doença renal crônica) nas pessoas com diagnóstico de hipertensão arterial. Controlar todos os riscos cardiovasculares identificados de acordo com padrões nacionais ou internacionais reconhecidos ${ }^{58,59}$.

- A atenção baseada em trabalho de equipe melhora o tratamento e o controle da hipertensão arterial ${ }^{34,60}$. Otimizar a atenção centrada no paciente com uso de abordagem baseada em trabalho de equipe para medir a pressão arterial, manter contato periódico com o paciente e realizar outras tarefas de rotina dentro do campo de regulamentação da atividade. Além da abordagem baseada em trabalho de equipe, com atuação de profissionais não médicos (funcionários administrativos do ambulatório, enfermeiros, farmacêuticos, agentes comunitários de saúde, etc.) de acordo com sua formação profissional, os funcionários administrativos, cônjuges e amigos podem ter uma participação importante na melhoria da efetividade do tratamento. Entretanto, o paciente sempre deve estar no centro da equipe.

- Avaliar cada paciente para verificar se é adequado o uso de um algoritmo/protocolo simples de manejo da hipertensão arterial para diagnóstico ou tratamento e individualizar o manejo na pequena parcela de pacientes para os quais o algoritmo é inadequado ou que apresentam efeitos colaterais ou intolerância ao medicamento ${ }^{61}$.

- Tratar pessoas com pressão arterial igual ou superior a 160/100 mmHg imediatamente após o diagnóstico com modificação do estilo de vida e medicamentos anti-hipertensivos.

- Exigir o uso de medicamentos anti-hipertensivos de alta qualidade, com ênfase naqueles que atendam a padrões aceitáveis de qualidade, tenham ação prolongada, possam ser tomados uma vez ao dia e estejam disponíveis continuamente para evitar a confusão e interrupção do tratamento que ocorrem nos casos de troca de medicamentos (mesmo quando clinicamente equivalentes)

- Avaliar periodicamente a adesão do paciente ao tratamento da hipertensão arterial.

- Usar registros com relatório de desempenho.

- Avaliar alimentação, tabagismo, consumo de álcool, atividade física e obesidade e oferecer orientação individualizada sobre o estilo de vida. Especificamente, aconselhar e ajudar 
todos os tabagistas a abandonarem o uso e aconselhar e ajudar todos aqueles que consomem álcool em excesso a limitarem ou interromperem o consumo.

- Incentivar e ajudar programas de comunitários de rastreamento da hipertensão arterial, como o Maio, Mês de Medição e o Dia Mundial da Hipertensão (17 de maio).

- Orientar os pacientes, as famílias dos pacientes e a população em geral.

\subsection{Atenção primária}

- Garantir que as organizações que representam os profissionais da atenção primária à saúde que fazem a prevenção, o diagnóstico e o tratamento da hipertensão arterial, sejam primordiais nas estratégias de controle da hipertensão arterial.

- Com as organizações de atenção primária, assegurar que haja diretrizes de tratamento da hipertensão arterial, na atenção primária adaptadas à população do país. Converter as diretrizes em algoritmos e protocolos de diagnóstico e tratamento simples e fáceis de usar, que incluam terapias de combinação em comprimido único ${ }^{46,61,62}$.

- Com as organizações de atenção primária, desenvolver programas educacionais padronizados com enfoque nas intervenções-chave para manejo e controle sistemático da hipertensão na atenção primária ${ }^{63}$.

- Quando viável, usar o progresso tecnológico (sistemas de saúde digitais e móveis) para engajar e empoderar os pacientes $^{64-66}$.

- O monitoramento domiciliar da pressão arterial (PA) combinado ao uso de telemetria para transmitir os valores registrados em casa ao profissional de saúde, bem como a resposta do profissional de saúde, ajuda a otimizar o tratamento, melhorar a adesão do paciente e reduzir a inércia terapêutica.

- Os smartphones com aplicativos relacionados ao manejo da hipertensão podem ser recursos de telemedicina de baixo custo no dia a dia. A Sociedade Europeia de Hipertensão dispõe de um aplicativo para smartphone (ESH CARE) validado e atualizado continuamente ${ }^{65,67-69}$.

\subsection{Reconhecimento e recompensa}

- Indicar programas, representantes de programa e indivíduos para o recebimento de prêmios da Liga Mundial de Hipertensão e da Sociedade Internacional de Hipertensão em reconhecimento pela redução do sal para a população e pelo controle da pressão arterial (disponíveis em inglês em: http://www. whleague.org/index.php/news-awards-recognition, consultado em 17 de abril de 2019; http:/ /ish-world.com/activities/awards-prizes.htm, consultado em 5 de maio de 2019).

\subsection{Promoção da causa}

- Defender políticas públicas saudáveis; principalmente aquelas que reduzem a quantidade de sal/sódio na alimentação, promovem dietas saudáveis e atividade física, reduzem o consumo prejudicial de álcool e eliminam o tabagismo e as gorduras trans artificiais ${ }^{31}$.

- Defender e promover a atualização das relações nacionais de medicamentos para assegurar a disponibilidade e acessibilidade de medicamentos anti-hipertensivos de alta qualidade, com ênfase em combinações de ação prolongada em comprido único.

- Defender políticas que incluam a regulamentação para assegurar a compra, a venda e o uso de monitores de pressão arterial automáticos validados, apropriados, exatos e braçadeiras ${ }^{70,71}$.

- Defender o treinamento periódico e a certificação dos profissionais de saúde e leigos que fazem a avaliação periódica da pressão arterial ${ }^{72}$.

- Defender a atenção primária centrada no paciente, colaborativa e baseada em trabalho de equipe ${ }^{50}$.

- Defender o uso de registros de hipertensão para seguimento clínico e feedback sobre o desempenho em ambulatórios que cuidam de pessoas hipertensas ${ }^{49,73,74}$.

- Defender o monitoramento e avaliação padronizados dos esforços de prevenção e controle da hipertensão arterial ${ }^{49,74-76}$.

- Acompanhar relatórios periódicos (trimestrais ou anuais) das taxas de controle de hipertensão arterial no país, além de defender e apoiar esforços para melhorar essas taxas.

\subsection{Empoderamento das pessoas}

- Usar como base da alimentação uma variedade de hortaliças (principalmente folhas verdes), frutas, frutos oleaginosos, sementes e leguminosas ${ }^{77}$.

- Consumir com maior frequência alimentos não processados ou minimamente processados.

- Escolher opções com baixo teor de sódio e não adicionar sal aos alimentos.

- Não fumar.

- Praticar atividade física.

- Alcançar e manter um peso saudável.

- Limitar o consumo de bebidas alcoólicas e não ultrapassar as recomendações de consumo diário e semanal máximo.

- Verificar a pressão arterial periodicamente e saber qual é o valor pressórico recomendado.

- Medir a sua própria pressão arterial. Aprender como obter medidas corretas da PA como usá-las, em parceria com a equipe de atenção à saúde.

- Defender políticas públicas saudáveis.

- Exigir o uso de medicamentos anti-hipertensivos de alta qualidade, com destaque para os de ação prolongada.

- Comunicar-se com o profissional de saúde por meio de ferramentas digitais e móveis de saúde validadas.

- Tomar regularmente os medicamentos eventualmente prescritos para tratamento da hipertensão arterial, até que sejam modificados por um profissional de saúde. Criar uma rotina para a tomada dos medicamentos e monitorar a ingestão dos comprimidos para assegurar a adesão.

\section{RECURSOS PARA BOAS PRÁTICAS MUNDIAIS}

- Recursos da OMS e RTSL para melhorar o controle da hipertensão estão disponíveis no Portal Conhecimentos e Ação, sobre doenças não transmissíveis (em espanhol em: https:/ / www.knowledge-action-portal.com/es/node, consultado em 17 de abril de 2019), no portal LINKS - Comunidade global para a saúde cardiovascular (em inglês em: https: / / www. linkscommunity.org / consultado em 17 de abril de 2019) e no site https://www.who.int/cardiovascular_diseases/hearts/ en/ (em inglês, consultado em 3 de junho de 2019).

- Recursos educacionais e de treinamento para prevenção e manejo da hipertensão também estão disponíveis no site da Organização Pan-Americana da Saúde (em espanhol em: 
https:/ / www.paho.org/hq/index.php?option=com_content\&view =article\&id=15056:hearts-in-the-americas\&Itemid=3465\&lang=es, consultado em 3 de junho de 2019) e no Campus Virtual de Saúde Pública (https://www.campusvirtualsp.org/pt-br/user/login, consultado em 6 de maio de 2019)

- Recursos da Liga Mundial de Hipertensão estão disponíveis em inglês em http:/ / www.whleague.org/ (consultado em 6 de maio de 2019

- Recursos da Sociedade Internacional de Hipertensão estão disponíveis em inglês em http://ish-world.com/index.htm (consultado em 6 de maio de 2019)

Este artigo informativo e chamada à ação é apoiado pela Liga Mundial de Hipertensão, Sociedade Argentina de Hipertensão, Escritório Regional da Liga Mundial de Hipertensão para a Ásia e o Pacífico, Escritório Regional Sudoeste Asiático da Liga Mundial de Hipertensão, Aliança Universitária de Pesquisa Africana, Comitê Belga de Hipertensão, Blue Cross e Blue Shield da Louisiana, Sociedade Brasileira de Hipertensão, Liga Búlgara de Hipertensão, Caja Costarricense de Seguro Social (Fundo de Seguro Social da Costa Rica), Fundação do Coração de Camarões, Liga Chinesa de Hipertensão, Colegas no cuidado, Hipertensão Canadá, Sociedade Europeia de Hipertensão, Conselho de Hipertensão da Sociedade Europeia de Cardiologia, Centro de Excelência para Doenças Não Transmissíveis de Ibadan, Sociedade Indiana de Hipertensão Arterial, Conselho Internacional de Prevenção e Reabilitação Cardiovascular, Sociedade Internacional para a Hipertensão Arterial, Sociedade Internacional para o Estudo da Hipertensão na Gravidez, Sociedade Internacional de Nefrologia, Fundação do Coração do Irã, Grupo Comissão Lancet de Hipertensão, Sociedade Latino-americana de Nefrologia e Hipertensão, Sociedade Letã de Hipertensão e Aterosclerose, Sociedade Mongol de Hipertensão, Comitê Técnico Assessor Nacional do Programa de Hipertensão Arterial de Cuba (Ministério de Saúde Pública), NHMRC Centro de Excelência em Pesquisa para Melhoria de Desfechos Cardiovasculares (Austrália), Fundação Onom (Mongólia), Sociedade Filipina de Hipertensão, Iniciativa Resolve to Save Lives, Sociedade Saudita de Gestão de Hipertensão, Sociedade Portuguesa de Hipertensão, Escritório Regional da Liga Mundial de Hipertensão para o Sudeste Asiático, Iniciativa de Inovações para
Controle do Acidente Vascular Cerebral da Nigéria, Pesquisa Investigativa e Rede Educacional sobre Acidente Vascular Cerebral (SIREN), Sociedade Sudanesa de Hipertensão, Sociedade Sueca de Hipertensão, Acidente Vascular Cerebral e Medicina Vascular, Rede de Suporte de Acidente Vascular Cerebral-Ghana, Sociedade de Hipertensão de Taiwan, Sociedade de Cardiologia de Taiwan, Sociedade Tailandesa de Hipertensão, Escola de Enfermagem de Ribeirão Preto da Universidade de São Paulo, Grupo de Ação Mundial de Hipertensão, Cimeira Mundial da Saúde, Organização Mundial de Acidente Vascular Cerebral.

Conflito de interesses. NRCC atuou como consultor remunerado da Fundação Novartis (2016-2017) para apoiar seu programa de melhoria do controle da hipertensão arterial em países de baixa e média renda, incluindo ajuda para viagens de visita ao local e contrato para desenvolver a pesquisa. NRCC prestou consultoria remunerada sobre avaliação correta da pressão arterial à Midway Corporation (2017) e é membro não remunerado da Ação Mundial sobre Sal e Saúde (WASH, na sigla em inglês). AES recebeu honorários de Novartis e Omron, por palestras científicas sobre pressão arterial e avaliação de risco, e da Servier, por apresentação sobre aumento da conscientização para medida da pressão arterial. Ela é consultora remunerada de Abbott Pharmaceuticals para medicamentos anti-hipertensivos e é presidente da Sociedade Internacional de Hipertensão. MAW foi consultor de Abbvie e Bristol Myers Squibb sobre segurança de medicamentos e recebeu subvenção para viagem da Omron. Ele é membro dos conselhos consultivos de Medtronic, ReCor e Ablative Solutions. MO declara ter recebido honorários por palestras para a Glaxo Smith Kline (GSK) e Takeda Inc. GP declara ter recebido honorários da Omron Health Care, Sanofi e Servier. AR declara ter recebido apoio para congressos da Servier. PW, LAB, PO, XHZ, CV, TK, DL, MJ, AM, FLP, MB, EB e JS não têm conflitos de interesse financeiro a declarar.

Declaração. As opiniões expressas no manuscrito são de responsabilidade exclusiva dos autores e não refletem necessariamente a opinião ou política da RPSP/PAJPH ou da Organização Pan-Americana da Saúde ou da Organização Mundial da Saúde.

\section{REFERENCIAS}

1. Mills KT, Bundy JD, Kelly TN, et al. Global Disparities of Hypertension Prevalence and Control: A Systematic Analysis of Population-Based Studies From 90 Countries. Circulation. 2016;134(6):441-450.

2. Vasan RS, Beiser A, Seshadri S, et al. Residual Lifetime Risk for Developing Hypertension in Middle-aged Women and Men. JAMA. 2002;287(8):1003-1010.

3. Organização Mundial da Saúde. A global brief on hypertension: silent killer, global public health crisis. World Health Day 2013. Genebra: OMS; 2013.

4. Muntner P, Carey RM, Gidding S, et al. Potential U.S. Population Impact of the 2017 American College of Cardiology/American Heart Association High Blood Pressure Guideline. J Am Coll Cardiol. 2018;71(2):109-118.
5. Wang JG, Liu L. Global Impact of 2017 American College of Cardiology/American Heart Association Hypertension Guidelines: A Perspective From China. Circulation. 2018;137(6):546-548.

6. Global Burden of Disease Website. Institute for Health Metrics and Evaluation. Disponível em inglês em: http://vizhub.healthdata. org/gbd-compare/, consultado em 13 de julho de 2019.

7. Forouzanfar MH, Liu P, Roth GA, et al. Global Burden of Hypertension and Systolic Blood Pressure of at Least 110 to $115 \mathrm{~mm} \mathrm{Hg}$, 1990-2015. JAMA. 2017;317(2):165-182.

8. Levy D, Larson MG, Vasan RS, Kannel WB, Ho KKL. The Progression From Hypertension to Congestive Heart Failure. JAMA. 1996;275(20):1557-1562

9. Feigin VL, Roth GA, Naghavi M, et al. Global burden of stroke and risk factors in 188 countries, during 1990\&\#x2013;2013: a systematic 
analysis for the Global Burden of Disease Study 2013. Lancet Neurol. 2016;15(9):913-924.

10. O'Donnell MJ, Chin SL, Rangarajan S, et al. Global and regional effects of potentially modifiable risk factors associated with acute stroke in 32 countries (INTERSTROKE): a case-control study. Lancet. 2016;388(10046):761-775.

11. Chen G, McAlister FA, Walker RL, Hemmelgarn BR, Campbell NR. Cardiovascular outcomes in framingham participants with diabetes: the importance of blood pressure. Hypertension. 2011;57(5):891-897.

12. Khan KS, Wojdyla D, Say L, Gulmezoglu AM, Van Look PFA. WHO analysis of causes of maternal death: a systematic review. Lancet. 2006;367(9516):1066-1074.

13. Levi MN, Macquin-Mavier I, Tropeano AI, Bachoud-Levi AC, Maison P. Antihypertensive classes, cognitive decline and incidence of dementia: a network meta-analysis. J Hypertens. 2013;31(6):1073-1082.

14. Udani S, Lazich I, Bakris GL. Epidemiology of hypertensive kidney disease. Nat Rev Nephrol. 2011;7(1):11-21.

15. Worldwide trends in blood pressure from 1975 to 2015: a pooled analysis of 1479 population-based measurement studies with 19.1 million participants. Lancet. 2017;389(10064):37-55.

16. Gaziano TA, Bitton A, Anand S, Weinstein MC. The global cost of nonoptimal blood pressure. Journal of Hypertension. 2009;27(7):1472-1477.

17. Organização Mundial da Saúde. Salvar vidas, reducir el gasto: Una respuesta estratégica a las enfermedades no transmisibles. Genebra: OMS; 2018 (WHO/NMH/NVI/18.8). Licença: CC BY-NC-SA 3.0 IGO.

18. Institute of Medicine of the National Academies. A Population-Based Policy and Systems Change Approach to Prevent and Control Hypertension-Brief Report. Washington, D.C., EUA: National Academy Press; 2010.

19. Joffres M, Campbell NR, Manns B, Tu K. Estimate of the benefits of a population-based reduction in dietary sodium additives on hypertension and its related health care costs in Canada. Can J Cardiol. 2007;23(6):437-443.

20. Puska P, Iacono JM, Nissinen A, et al. Dietary fat and blood pressure: an intervention study on the effects of a low-fat diet with two levels of polyunsaturated fat. Prev Med. 1985;14(5):573-584.

21. Leng B, Jin Y, Li G, Chen L, Jin N. Socioeconomic status and hypertension: a meta-analysis. J Hypertens. 2015;33(2):221-229.

22. Chor D, Pinho Ribeiro AL, Sa Carvalho M, et al. Prevalence, Awareness, Treatment and Influence of Socioeconomic Variables on Control of High Blood Pressure: Results of the ELSA-Brasil Study. PloS ONE. 2015;10(6):e0127382.

23. Liew SJ, Lee JT, Tan CS, Koh CHG, Van Dam R, Muller-Riemenschneider F. Sociodemographic factors in relation to hypertension prevalence, awareness, treatment and control in a multi-ethnic Asian population: a cross-sectional study. BMJ open. 2019;9(5):e025869.

24. Organização Mundial da Saúde. Global status report on noncommunicable disease 2014. Genebra: OMS; 2014. 9789241564854.

25. Beaney T, Burrell LM, Castillo RR, et al. May Measurement Month 2018: a pragmatic global screening campaign to raise awareness of blood pressure by the International Society of Hypertension. Eur Heart J. 2019; doi:10.1093/eurheartj/ehz300.

26. Torlasco C, Faini A, Makil E, et al. Cardiovascular risk and hypertension control in Italy. Data from the 2015 World Hypertension Day. Int J Cardiol. 2017;243:529-532.

27. Ataklte F, Erqou S, Kaptoge S, Taye B, Echouffo-Tcheugui JB, Kengne AP. Burden of Undiagnosed Hypertension in Sub-Saharan Africa: A Systematic Review and Meta-Analysis. Hypertension. 2015:291-298.

28. Masters R, Anwar E, Collins B, Cookson R, Capewell S. Return on investment of public health interventions: a systematic review. J Epidemiol Community Health. 2017;71(8):827-834.

29. Fórum Econômico Mundial e Escola de Saúde Pública de Harvard, Organização Mundial da Saúde. From Burden to "Best Buys": Reducing the Economic Impact of Non-Communicable Diseases in Low- and Middle-Income Countries. Genebra: : Fórum Econômico Mundial; 2011.

30. Nugent R. Benefits and Costs of the Non-Communicable Disease Targets for the Post-2015 Development Agenda. Copenhagen Consensus Center. 2015:I-II, 1-25.

31. Organização Mundial da Saúde. WHO Global Action Plan for the Prevention and Control of Noncommunicable Diseases 2013-2020. Genebra: OMS; 2013.
32. Organização Mundial da Saúde. SHAKE the salt habit. The SHAKE Technical Package for Salt Reduction. Genebra: OMS;2016.

33. Organização Mundial da Saúde. Global strategy to reduce the harmful use of alcohol. Genebra: OMS; 2010.

34. Whelton PK, Carey RM, Aronow WS, et al. 2017 ACC/AHA/ AAPA / ABC / ACPM / AGS / APhA / ASH / ASPC / NMA / PCNA Guideline for the Prevention, Detection, Evaluation, and Management of High Blood Pressure in Adults: A Report of the American College of Cardiology/American Heart Association Task Force on Clinical Practice Guidelines. Hypertension. 2017;71:e13-e115.

35. McAlister FA, Robitaille C, Gillespie C, et al. The Impact of Cardiovascular Risk Factor Profiles on Blood Pressure Control Rates in Adults From Canada and the United States. Can I Cardiol. 2013;29:598-605.

36. Rantala AO, Kauma H, Lilja M, Savolainen MJ, Reunanen A. Prevalence of the metabolic syndrome in drug-treated hypertensive patients and control subjects. Journal of Internal Medicine. 1999;245:163-174.

37. Organização Mundial da Saúde. HEARTS: Technical package for cardiovascular disease management in primary health care. Genebra: OMS; 2016.

38. Williams B, Mancia G, Spiering W, et al. 2018 ESC/ESH Guidelines for the management of arterial hypertension. Eur Heart $J$. 2018;39(33):3021-3104.

39. Leung AA, Nerenberg K, Daskalopoulou SS, et al. Hypertension Canada's 2016 Canadian Hypertension Education Program Guidelines for Blood Pressure Measurement, Diagnosis, Assessment of Risk, Prevention, and Treatment of Hypertension. CanJ Cardiol. 2016;32(5):569-588.

40. Wright Jr JT, Fine LJ, Lackland DT, Ogedegbe G, Dennison Himmelfarb CR. Evidence Supporting a Systolic Blood Pressure Goal of Less Than $150 \mathrm{~mm} \mathrm{Hg}$ in Patients Aged 60 Years or Older: The Minority View. Annals of Internal Medicine. 2014;1(160):7-499.

41. Tsujimoto T, Kajio H. Benefits of Intensive Blood Pressure Treatment in Patients With Type 2 Diabetes Mellitus Receiving Standard but Not Intensive Glycemic Control. Hypertension. 2018;72(2):323-330.

42. Campbell NR, Lackland DT, Niebylski ML, World Hypertension L, International Society of Hypertension Executive C. High Blood Pressure: Why Prevention and Control are Urgent and Important-A 2014 Fact Sheet From the World Hypertension League and the International Society of Hypertension. J Clin Hypertens. 2014;16(8):551-553.

43. Campbell NR, Khalsa T, Lackland DT, et al. High Blood Pressure 2016: Why Prevention and Control Are Urgent and Important. The World Hypertension League, International Society of Hypertension, World Stroke Organization, International Diabetes Foundation, International Council of Cardiovascular Prevention and Rehabilitation, International Society of Nephrology. J Clin Hypertens (Greenwich). 2016;18(8):714-717.

44. Bennett JE, Stevens GA, Mathers CD, et al. NCD Countdown 2030: worldwide trends in non-communicable disease mortality and progress towards Sustainable Development Goal target 3.4. Lancet. 2018;392(10152):1072-1088.

45. Kotchen TA. The search for strategies to control hypertension. Circulation. 2010;122(12):1141-1143.

46. Organização Mundial da Saúde. HEARTS Paquete técnico para el manejo de las enfermedades cardiovasculares en la atencion primaria de salud. Evidencia: Protocolos de tratamiento clinico basados en la evidencia. Genebra: OMS; 2017

47. Organização Mundial da Saúde. HEARTS Paquete técnico para el manejo de las enfermedades cardiovasculares en la atención primaria de salud. Acceso a medicamentos y tecnologías esenciales. Genebra: OMS; 2018.

48. Organização Mundial da Saúde. HEARTS Paquete técnico para el manejo de las enfermedades cardiovasculares en la atención primaria de salud. Hábitos y estilos de vida saludables: asesoramiento para los pacientes. Genebra: OMS; 2018

49. Organização Mundial da Saúde. HEARTS Technical package for cardiovascular disease management in primary health care: systems for monitoring. Genebra: OMS;2018 (WHO/NMH/NVI/18.5). Licença: CC BY-NC-SA 3.0 IGO. Disponíveis em http://www.who.int/ cardiovascular_diseases/hearts/en/.

50. Organização Mundial da Saúde. HEARTS Paquete técnico para el manejo de las enfermedades cardiovasculares en la atención primaria de salud. Trabajo basado en equipos multidisciplinarios. Genebra: OMS; 2018. 
51. Frieden TR, Jaffe MG. Saving 100 million lives by improving global treatment of hypertension and reducing cardiovascular disease risk factors. J Clin Hypertens (Greenwich). 2018;20:208-211.

52. Kaczorowski J, Chambers LW, Dolovich L, et al. Improving cardiovascular health at population level: 39 community cluster randomised trial of Cardiovascular Health Awareness Program (CHAP). BMJ. 2011;342:d442.

53. Ferrari P. Reasons for therapeutic inertia when managing hypertension in clinical practice in non-Western countries. J Hum Hypertens. 2008; DOI:10.1038/jhh.2008.117.

54. Okonofua EC, Simpson KN, Jesri A, Rehman SU, Durkalski VL, Egan BM. Therapeutic Inertia Is an Impediment to Achieving the Healthy People 2010 Blood Pressure Control Goals. Hypertension. 2006;47(3):345-351.

55. Mangat BK, Campbell N, Mohan S, et al. Resources for Blood Pressure Screening Programs in Low Resource Settings: A Guide From the World Hypertension League. J Clin Hypertens (Greenwich). 2015;17(6):418-420.

56. Campbell N, Touyz R, Lackland D, Redburn K, Niebylski M. Celebrate World Hypertension Day (WHD) on May 17, 2015, and Contribute to Improving Awareness of Hypertension. J Clin Hypertens (Greenwich). 2015;17(4):317-318.

57. Campbell NC, Lackland DT, Lisheng L, et al. The World Hypertension League: A Look Back and a Vision Forward. J Clin Hypertens (Greenwich). 2015;17(1):5-6.

58. Thomopoulos C, Parati G, Zanchetti A. Effects of blood pressure lowering treatment in hypertension: 8 . Outcome reductions vs. discontinuations because of adverse drug events - meta-analyses of randomized trials. J Hypertens. 2016;34(8):1451-1463.

59. Organização Mundial da Saúde. HEARTS Technical package for cardiovascular disease management in primary health care. Genebra: OMS; 2018.

60. Mills KT, Obst KM, Shen W, et al. Comparative Effectiveness of Implementation Strategies for Blood Pressure Control in Hypertensive Patients: A Systematic Review and Meta-analysis. Ann Intern Med. 2018;168(2):110-120.

61. Frieden TR, King SM, Wright JS. Protocol-based treatment of hypertension: a critical step on the pathway to progress. JAMA. 2014;311(1):21-22.

62. Organização Mundial da Saúde. HEARTS Technical package for cardiovascular disease management in primary health care: Implementation guide. Genebra: OMS; 2018 (WHO/NMH/NVI/18.14). Licença: CC BY-NC-SA 3.0 IGO.

63. Campbell N, Tobe S. The Canadian effort to prevent and control hypertension. Can other countries adopt Canadian strategies? Curr Opin Cardiol. 2010;25(4):366-372.

64. Parati G, Stergiou GS, Asmar R, et al. European Society of Hypertension Practice Guidelines for home blood pressure monitoring. J Hum Hypertens. 2010:1-7.

65. Parati G, Omboni S, Albini F, et al. Home blood pressure telemonitoring improves hypertension control in general practice. The TeleBPCare study. J Hypertens. 2009;27(1):198-203.

66. Albini F, Xiaoqiu L, Torlasco C, et al. An ICT and mobile health integrated approach to optimize patients' education on hypertension and its management by physicians: The Patients Optimal Strategy of Treatment(POST) pilot study. Conference proceedings: Annual
International Conference of the IEEE Engineering in Medicine and Biology Society IEEE Engineering in Medicine and Biology Society Annual Conference 2016;2016:517-520.

67. Widmer RJ, Collins NM, Collins CS, West CP, Lerman LO, Lerman A. Digital Health Interventions for the Prevention of Cardiovascular Disease: A Systematic Review and Meta-analysis. Mayo Clin Proc. 2015;90(4):469-480.

68. Parati G, Stergiou GS, Asmar R, et al. European Society of Hypertension guidelines for blood pressure monitoring at home: a summary report of the Second International Consensus Conference on Home Blood Pressure Monitoring. J Hypertens. 2008;26(8):1505-1526.

69. Burke LE, Ma J, Azar KM, et al. Current Science on Consumer Use of Mobile Health for Cardiovascular Disease Prevention: A Scientific Statement From the American Heart Association. Circulation. 2015;132(12):1157-1213.

70. Campbell NR, Berbari AE, Cloutier L, et al. Policy Statement of the World Hypertension League on Noninvasive Blood Pressure Measurement Devices and Blood Pressure Measurement in the Clinical or Community Setting. J Clin Hypertens (Greenwich). 2014;16(5):320-322.

71. Campbell NR, Gelfer M, Stergiou GS, et al. A Call to Regulate Manufacture and Marketing of Blood Pressure Devices and Cuffs. A Position Statement From the World Hypertension League, International Society of Hypertension and Supporting Hypertension Organizations. J Clin Hypertens. 2016;18(5):378-380.

72. Padwal R, Campbell NRC, Schutte AE, et al. Optimizing observer performance of clinic blood pressure measurement: a position statement from the Lancet Commission on Hypertension Group. J Hypertension. 2019; DOI:10.1097/HJH.0000000000002112.

73. Campbell N, Ordunez P, Jaffe MG, et al. Implementing standardized performance indicators to improve hypertension control at both the population and healthcare organization levels. J Clin Hypertens (Greenwich). 2017;19(5):456-461.

74. Campbell NRC, Ordunez P, DiPette DJ, et al. Monitoring and evaluation framework for hypertension programs. A collaboration between the Pan American Health Organization and World Hypertension League. J Clin Hypertens (Greenwich). 2018;DOI:10.111/ JCH.13307.

75. Campbell NR, McAlister FA, Quan H. Monitoring and Evaluating Efforts to Control Hypertension in Canada: Why, How, and What It Tells Us Needs to Be Done About Current Care Gaps. Can J Cardiol. 2013;29:564-570.

76. Benjamin EJ, Muntner P, Alonso A, et al. Heart Disease and Stroke Statistics-2019 Update: A Report From the American Heart Association. Circulation. 2019;139(10):e56-e66.

77. Owolabi MO, Sarfo F, Akinyemi R, et al. Dominant modifiable risk factors for stroke in Ghana and Nigeria (SIREN): a case-control study. Lancet Glob Health. 2018;6(4):e436-e446.

Manuscrito original em inglês recebido em 25 de setembro de 2019, aceito em 5 de novembro de 2019. 


\section{São Paulo call to action for the prevention and control of high blood pressure: 2020}

ABSTRACT - About 1/4th of adults have high blood pressure which is the single most important risk for death (including heart disease and stroke).

- There are effective policies that could facilitate people making healthy choices to prevent raised blood pressure, and if fully implemented, could largely prevent hypertension from occurring.

- Hypertension is easy to screen and treat for BUT only about $50 \%$ of adults with hypertension are aware of their condition and only about 1 in 7 is adequately treated.

- Preventing and controlling high blood pressure is the major mechanism for NCD prevention and control and a model for other NCD risks.

- Effective lifestyle and drug treatments could prevent and control hypertension in most individuals if systematically applied to the population, simple interventions are feasible in all settings, and can be used to enhance primary care.

- Urgent sustained action is needed is needed for effective public policies and health system changes to prevent and control hypertension.

Keywords

Hypertension; cardiovascular diseases; global health; diet, sodium-restricted; stroke.

\section{Llamado a la acción de San Pablo para la prevención y el control de la hipertensión arterial, 2020}

RESUMEN

Palabras clave
- Cerca de una cuarta parte de los adultos tienen hipertensión, el principal factor de riesgo de muerte (inclusive la causada por cardiopatía y accidente cerebrovascular).

- Existen políticas eficaces que podrían ayudar a las personas a elegir opciones saludables para prevenir el aumento de la presión arterial; si se las aplicara plenamente, se podría evitar en gran medida el desarrollo de hipertensión.

- La hipertensión es fácil de detectar y tratar, PERO solo alrededor de 50\% de los adultos que presentan dicha afección son conscientes de su situación y solamente 1 de cada 7 de ellos recibe el tratamiento adecuado.

- La prevención y el control de la hipertensión es el mecanismo principal para prevenir y controlar las enfermedades no transmisibles y un modelo para evitar otros riesgos de presentar dichas enfermedades.

- La adopción de un modo de vida saludable y el tratamiento farmacológico efectivo podrían prevenir y controlar la hipertensión en la mayoría de las personas si se implementaran de manera sistemática en la población; en todos los entornos es posible aplicar intervenciones sencillas, que pueden usarse para mejorar la atención primaria.

- Es urgente adoptar medidas sostenidas para introducir cambios eficaces en las políticas públicas y los sistemas de salud pública con miras a prevenir y controlar la hipertensión.

Hipertensión; enfermedades cardiovasculares; salud global; dieta hiposódica; accidente cerebrovascular. 